\title{
Paradoxical effects of repeat interruptions on spinocerebellar ataxia type 10 expansions and repeat instability
}

\author{
Karen N McFarland ${ }^{1,2,10}$, Jilin Liu ${ }^{1,2,10}$, Ivette Landrian ${ }^{1,2}$, Rui Gao ${ }^{3}$, Partha S Sarkar ${ }^{3}$, Salmo Raskin ${ }^{4}$, \\ Mariana Moscovich ${ }^{1,2,5}$, Emilia M Gatto ${ }^{6,7}$, Hélio A G Teive ${ }^{5}$, Adriana Ochoa ${ }^{8}$, Astrid Rasmussen ${ }^{9}$ and \\ Tetsuo Ashizawa ${ }^{*, 1,2}$
}

Spinocerebellar ataxia type 10 (SCA10) is an autosomal dominant neurodegenerative disorder caused by a noncoding ATTCT pentanucleotide expansion. An inverse correlation between SCA10 expansion size and age at onset has been reported, and genetic anticipation has been documented. Interruptions in the ATTCT expansion are known to occur within the expansion. In order to determine the effect of repeat interruptions in SCA10 expansions, we designed a PCR assay to easily identify ATCCT repeat interruptions in the $5^{\prime}$-end of the expansion. We screened a cohort of 31 SCA10 families of Mexican, Brazilian and Argentinean ancestry to identify those with ATCCT repeat interruptions within their SCA10 expansions. We then studied the effects of ATCCT interruptions on intergenerational repeat instability, anticipation and age at onset. We find that the SCA10 expansion size is larger in SCA10 patients with an interrupted allele, but there is no difference in the age at onset compared with those expansions without detectable interruptions. An inverse correlation between the expansion size and the age at onset was found only with SCA10 alleles without interruptions. Interrupted expansion alleles show anticipation but are accompanied by a paradoxical contraction in intergenerational repeat size. In conclusion, we find that SCA10 expansions with ATCCT interruptions dramatically differ from SCA10 expansions without detectable ATCCT interruptions in repeat-size-instability dynamics and pathogenicity.

European Journal of Human Genetics (2013) 21, 1272-1276; doi:10.1038/ejhg.2013.32; published online 27 February 2013

Keywords: repeat instability; repeat interruptions; anticipation

\section{INTRODUCTION}

Spinocerebellar ataxia type 10 (SCA10 (MIM ID \#603516)), an autosomal dominant, neurodegenerative disorder, typically presents with progressive ataxia, which may be accompanied by ethnicitydependent occurrences of epileptic seizures ${ }^{1-5}$ and variable extracerebellar symptoms such as hyperreflexia, polyneuropathy, low IQ and neuropsychiatric symptoms. ${ }^{1}$ Genetic anticipation is a noted disease feature. ${ }^{4,6}$

SCA10 results from a noncoding pentanucleotide repeat (ATTCT) expansion within intron 9 of Ataxin 10. ${ }^{7}$ Normally ranging from 9 to 32 repeats, the ATTCT repeat expands up to 4500 repeats in SCA10 patients. $^{7-9}$ Past analysis on patients of one SCA10 family revealed that the $5^{\prime}$-end of the expansion contained ATATTCT and ATTTTCT heptanucleotide interruptions, disrupting the long ATTCT repeat, whereas the SCA10 expansion in members of a second family lacked these interruptions. ${ }^{8}$ Further investigations of the effect of these repeat interruptions have been hampered by laborious identification of repeat interruptions by end sequencing of the SCA10 expansion.
To examine the effects of the repeat interruption in SCA10 expansion alleles, we developed a novel PCR assay to quickly identify the repeat interruptions. We then applied this assay to a large cohort of SCA10 families to study the effect of repeat interruptions on expansion size, repeat instability, age at onset and anticipation.

\section{MATERIALS AND METHODS \\ DNA samples}

Blood samples were obtained from 298 members of 31 SCA10 families, including 18 Mexican families, 9 Brazilian families and 1 Argentinean family, after obtaining informed consent under protocols approved by local institutional review boards. Some of these families have been previously described. ${ }^{1-4,10}$ Genomic DNA was extracted from peripheral blood leukocytes using conventional methods.

$3^{\prime}$ end sequence from the SCA10 expansion Genomic DNA isolated from SCA10 somatic cell hybrid lines ${ }^{11}$ was used as template in a long-range PCR using HotStarTaq Master Mix (Qiagen,

${ }^{1}$ Department of Neurology, University of Florida, Gainesville, FL, USA: ${ }^{2}$ Evelyn F \& William L McKnight Brain Institute, University of Florida, Gainesville, FL, USA; ${ }^{3}$ Department of Neurology, University of Texas Medical Branch, Galveston, TX, USA; ${ }^{4}$ Core for Advanced Molecular Investigation, Graduate Program in Health Sciences, Center for Biological and Health Sciences, Pontifical Catholic University of Paraná, Curitiba, Brazil; ${ }^{5}$ Movement Disorders Unit, Neurology Service, Hospital de Clínicas, Federal University of Paraná, Curitiba, Brazil; ${ }^{6}$ Departamento de Neurología, Sanatorio de la Trinidad Mitre, Buenos Aires, Argentina; ${ }^{7}$ Department of Movement Disorders, Instituto de Neurosciencias Buenos Aires (INEBA), Buenos Aires, Argentina; ${ }^{8}$ Department of Neurogenetics, Instituto Nacional de Neurologia y Neurocirugia Manuel Velasco Suarez, Mexico City, Mexico; ${ }^{9}$ Arthritis and Clinical Immunology Research Program, Oklahoma Medical Research Foundation, Oklahoma City, OK, USA

10These authors contributed equally to this work.

*Correspondence: Dr T Ashizawa, Department of Neurology, University of Florida, 1149 S. Newell Dr, L3-100, Gainesville, FL 32611, USA. Tel: + 1352 273 5550; Fax: + 1352 273 5575; E-mail; tetsuo.ashizawa@neurology.ufl.edu

Received 3 October 2012; revised 2 January 2013; accepted 30 January 2013; published online 27 February 2013 
Germantown, MD, USA) with forward (5'-GTCATCAGGTTGTTGCTGTGGA AAGGGGCGGTA- $\left.3^{\prime}\right)$ and reverse $\left(5^{\prime}\right.$-GCCACTGCACTCCAGCCTGGGC AACATAGAGAG- $3^{\prime}$ ) primers flanking the repeat. PCR products were cloned into the TOPO-TA vector (Invitrogen, Grand Island, NY, USA) and propagated in Escherichia coli grown at $25^{\circ} \mathrm{C}$. Sixteen transformants were selected and sequenced on an ABI Prism 377 Genetic Analyzer (Applied Biosystems, Grand Island, NY, USA) using primers specific to unique, non-repeat sequences flanking the SCA10 expansion.

\section{Identification of SCA10-positive individuals}

Normal SCA10 alleles were identified by PCR as described. ${ }^{2,12}$ Samples containing a single normal allele were further analyzed by repeat-primed PCR (RP-PCR) as described. ${ }^{13}$ RP-PCR-positive samples were subjected to Southern blot analysis to determine the expansion size as previously described.

\section{ATCCT repeat interruption PCR (ATCCT-PCR)}

ATCCT reverse primer (L2RT: 5'-TACGCATCCCAGTTTGAGACGG $\left(\right.$ AGGAT) ${ }_{6}-3^{\prime}$ ) and forward primer (LP-L: $5^{\prime}$-GGAATTCGGCTTAAATATCCA ACTAAAAGACTACTAGAATGG- $3^{\prime}$ ) were paired in a PCR reaction using KOD Hot Start DNA Polymerase (EMD Millipore, Billerica, MA, USA). The 1.2-kb PCR products were gel purified (Qiagen) and used as template for sequence reactions on an ABI 3130 Genetic Analyzer (Applied Biosystems).

\section{Statistical analysis}

Prism 5 (Synergy Software, La Jolla, CA, USA) was used to generate graphs and perform statistical analyses. For comparisons of two groups, Student's $t$-test was performed. For comparisons of four groups, one-way analysis of variance with post-hoc Bonferroni's multiple comparison test was performed. Pearson's correlation coefficient was calculated for correlations of age at onset and expansion size. Two-tailed $P$-values were calculated. Standard deviation of the mean is expressed throughout.

\section{RESULTS}

Long-range PCR products across the SCA10 expansion (Figure 1a, primer combination LR-F and LR-R) were generated from the genomic DNA of somatic cell hybrid lines derived from SCA10 patients, ${ }^{11}$ subcloned in E. coli and then sequenced at the ends. We identified heptanucleotide (ATATTCT and ATTTTTCT) repeat interruptions from the $5^{\prime}$-end of the repeat, matching those previously identified ${ }^{8}$ (Figure 1b). In addition, we identified a novel ATCCT interruption located at the $3^{\prime}$-end of the expansion (Figure 1c).

We then designed an ATCCT repeat annealing primer for use in a PCR assay and paired it with an upstream primer that annealed to unique sequence outside the expansion (Figure 1a, primer combination LP-L and L2RT). With these primers, we obtained a 1.2-kb PCR product from some but not all SCA10-positive patients (Figure 1d). Direct sequencing of the purified PCR product revealed stretches of pure repeats frequently interrupted by a combination of heptanucleotide ATTTTCT and ATATTCT interruptions similar to what was seen at the $5^{\prime}$-end, in addition to the more distally located pentanucleotide ATCCT motifs (Figure 1e). As the reverse primer specifically anneals to the ATCCT motif, we refer to this PCR protocol as well as positive SCA10 patients as 'ATCCT-PCR' and 'ATCCT-positive', respectively. Consequently, 'ATCCT-positive' expansions are defined as containing

a

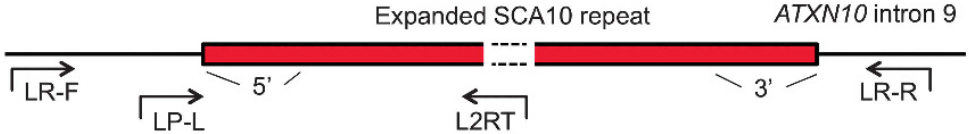

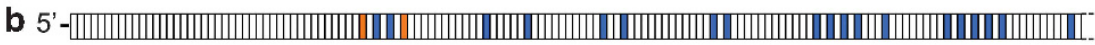

c

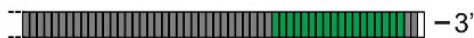

d

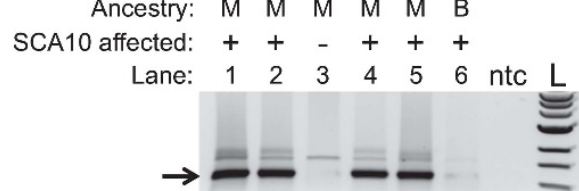

\begin{tabular}{|c|c|}
\hline Repeat Motif: & ATATTCT \\
\hline АТTCT & ATCCC \\
\hline АTTTTCT & ATCCT \\
\hline
\end{tabular}

e

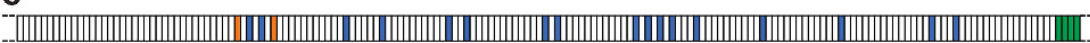

Figure 1 Configuration of ATCCT repeat interruptions within the SCA10 pentanucleotide expansion. (a) Schematic of the SCA10 repeat expansion (red rectangle) in intron 9 of the Ataxin 10 gene, depicting the $5^{\prime}$ - and $3^{\prime}$-ends of the repeat. Primers used to amplify full-length (LR-F and LR-R) and ATCCT-PCR (LP-L and L2RT) products are indicated by arrows relative to the repeat. (b) Schematic of the motif structure of the $5^{\prime}$-end of the expansion obtained from SCA10 hybrid cell lines. Sequence obtained is as follows: $5^{\prime}$-ATTCT ${ }_{40-41}$ ATTTTCT ATTCT (ATATTCT ATTCT) 2 ATTTTCT ATTCT 11 ATATTCT ATTCT $_{5}$ ATATTCT ATTCT 10 ATATTCT ATTCT 2 ATATTCT ATTCT 12 (ATATTCT ATTCT) 2 ATTCT 11 (ATATTCT ATTCT) 4 ATTCT 2 ATATTCT ATTCT $8-10$ (ATATTCT ATTCT) ${ }_{5}$ ATTCT $_{7-8}$ ATATTCT ATTCT $_{n}-3^{\prime}$ and matches previously identified interruption sequences. ${ }^{8}$ (c) Schematic of the motif structure of the $3^{\prime}$-end of the expansion obtained from SCA10 hybrid lines. Sequence obtained is as follows: $5^{\prime}$-(ATCCT) $34+(\text { ATCCC })_{4-28}$ (ATCCT) $)_{2}$ ATTCT ${ }_{1}-3^{\prime}$. (d) Typical ATCCT-PCR results from affected $(+)$ or unaffected $(-)$ samples of Mexican $(M)$ or Brazilian (B) ancestry. Lanes containing no template control (ntc) or DNA size standard $(\mathrm{L})$ are indicated. The arrow indicates the 1.2-kb ATCCT-PCR product size. The faint large $(\sim 2.2 \mathrm{~kb})$ band may be a product that resulted from annealing of the reverse primer to interrupted sequence further downstream, whereas the faint middle band is a constant background band found even in unaffected individuals. (e) An example of sequence obtained by direct sequencing of 1.2-kb band of the ATCCT-positive PCR product (from lane 1 above; $5^{\prime}$-ATTCT $_{30}$ ATTTTCT ATTCT (ATATTCT ATTCT) 2 ATTTTCT ATTCT 11 ATATTCT ATTCT 5 ATATTCT ATTCT 10 ATATTCT ATTCT 2 ATATTCT ATTCT 12 ATATTCT ATTCT ATATTCT ATTCT 12 (ATATTCT ATTCT) ${ }_{4}$ ATTCT $_{2}$ ATATTCT ATTCT 10 (ATATTCT ATTCT) $)_{5}$ ATTCT 7 ATATTCT ATTCT 14 ATATTCT ATTCT 3 ATATTCT ATTCT 16 $(\text { ATCCT })_{2+}-3^{\prime}$ ). An exact count of ATTCT repeats at the $5^{\prime}$-end cannot be made as the sequencing primer anneals near the beginning of the repeat and sequence data began with ATTCT sequences. However, at least 30-35 repeats were observed in various samples. In panels b, c and e: white rectangle, ATTCT motif; orange rectangle, ATTTTCT interruption motif; blue rectangle, ATATTCT interruption motif; gray rectangle, ATCCT interruption motif; green rectangle, ATCCC interruption motif as indicated. 
an ATCCT repeat 1.2-kb downstream of the forward primer annealing site. With this novel ATCCT-PCR assay, we can easily and quickly screen for the presence of ATCCT repeat interruptions within SCA10 expansions.

After screening individuals from SCA10 families for the presence of the expanded SCA10 allele by RP-PCR and determining the size of the expansion by Southern blotting, we then checked for the presence of ATCCT repeat interruptions by our new ATCCT-PCR protocol. We then, using family pedigrees, identified whether the allele was maternally or paternally transmitted. In our analysis, we also included published data from an additional 15 SCA10-positive individuals from the Mexican-American family ${ }^{6}$ that was originally identified as not carrying ATATTCT and ATTTTCT heptanucleotide interruptions within their SCA10 expansions. ${ }^{8}$

Individuals originally identified with heptanucleotide repeat interruptions $^{8}$ are positive for the ATCCT-PCR reaction. The ATCCT interruption was seen in all SCA10-positive family members of five families of Mexican origin. Furthermore, all SCA10-positive members of the nine Brazilian, the single Argentinean and the remaining thirteen Mexican families tested ATCCT negative. As negative controls, seven Brazilian, three Argentinean and 77 Mexican SCA10-negative samples were tested and determined to be ATCCT-negative as expected. In addition, in families where multiple members were tested, ATCCT-positive or -negative status was maintained within the family, including 16 parent-child pairs. The faithful heritability of these repeat interruptions was previously noted. ${ }^{8}$

We first examined the difference in expansion size between alleles with ATCCT interruption and those without (Figure 2a). We found that ATCCT-positive SCA10 alleles $(2757 \pm 835$ repeats, $n=32)$ are significantly longer $(t(108)=6.132, P<0.0001)$ than ATCCT-negative SCA10 alleles ( $1773 \pm 734$ repeats, $n=78)$.

We next compared the age at onset in patients with ATCCTpositive versus ATCCT-negative SCA10 expansions (Figure 2b). We found no significant difference (Welch-corrected $t(70)=0.8693$, $P=0.3877)$ in the mean age at onset in SCA10 patients with ATCCT-positive $(34.0 \pm 5.5$ years, $n=22)$ and ATCCT-negative (32.3 \pm 11.9 years, $n=51)$ SCA10 expansions. However, the variance between the two groups differed significantly $(F(50,21)=4.595$, $P=0.0003$ ).

We then identified 43 parent-child pairs (Supplementary Table 1), where we had obtained the size of the SCA10 expansion in our pedigrees. We calculated the intergenerational change in expansion size by subtracting the size of the parents' expansion from the size of the child's expansion (Figure 2c) and found a significant effect $(F(3,38)=17.97, P<0.0001)$. Here, differences were noted in the gender of the parent. Maternally transmitted expansions behaved similarly, whether ATCCT-negative $(-22 \pm 167$ repeats, $n=14)$ or ATCCT-positive expansions ( $54 \pm 116$ repeats, $n=6$ ), and were relatively stable. However, intergenerational instability in paternally transmitted alleles was dramatically different: ATCCT-positive expansions contracted $(-577 \pm 827$ repeats, $n=12)$, whereas ATCCT-negative expansions enlarged (1558 \pm 1091 repeats, $n=10)$. Significant differences were seen between paternally transmitted, ATCCT-negative and paternally transmitted, ATCCT-positive expansions $(P<0.0001)$. We also found a statistical difference between paternally transmitted, ATCCT-negative and maternally transmitted, ATCCT-negative alleles $(P<0.001)$, as well as between paternally transmitted, ATCCT-negative and maternally transmitted, ATCCTpositive $(P<0.01)$ alleles.

We next identified 36 parent-child pairs in our pedigrees where we had obtained the age at onset (Supplementary Table 1). We calculated
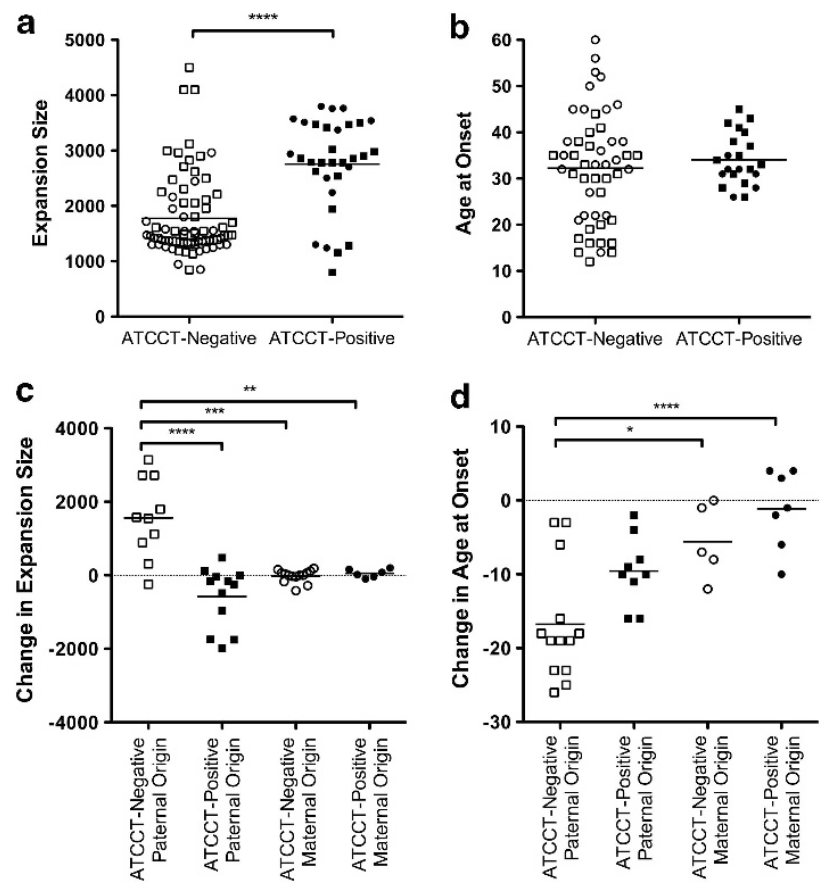

Figure 2 Effects of ATCCT interruptions on SCA10 expansions and age at onset. (a) Mean SCA10 expansion size in ATCCT-negative alleles (white squares, paternally transmitted, and circles, maternally transmitted) and ATCCT-positive alleles (black squares, paternally transmitted, and circles, maternally transmitted). (b) Mean age at onset, symbols as in panel a. (c) Mean intergenerational change in expansion size as calculated by subtracting the parents' expansion size from that of their childs' in paternally transmitted, ATCCT-negative alleles (white squares), paternally transmitted ATCCT-positive alleles (black squares), maternally transmitted, ATCCT-negative alleles (white circles) and maternally transmitted, ATCCT-positive alleles (black circles). (d) Mean intergenerational change in age at onset as calculated by subtracting the parent's age at onset from that of their child, symbols as in c. ${ }^{*} P<0.05 ;{ }^{*} P<0.01 ;{ }^{* *} P<0.001 ;{ }^{* * *} P<0.0001$.

the change in age at onset by subtracting the age at onset of the parent from the age at onset of the child (Figure $2 \mathrm{~d}$ ) and found a statistically significant effect on the change in age at onset $(F(3,30)=10.41$, $P=0.0002$ ). All four groups demonstrated anticipation with an earlier average age at onset in subsequent generations although the magnitude of the change differed between groups. Maternally transmitted alleles demonstrated the least amount of change in the age at onset between generations $(-1.1 \pm 5.4$ years in ATTCT-positive alleles, $n=9$, versus $-5.6 \pm 5.0$ years in ATTCT-negative alleles, $n=5$ ). Paternally transmitted alleles demonstrated marked anticipation. ATCCT-positive alleles had a mean change in age at onset of $-9.6 \pm 4.7$ years $(n=9)$, whereas ATCCT-negative alleles had a mean change in age at onset of $-16.8 \pm 7.9$ years $(n=13)$. Between these groups a significant difference in the change in age at onset was found between paternally and maternally transmitted ATCCT-negative alleles $(P<0.05)$ as well as between paternally transmitted, ATCCTnegative alleles and maternally transmitted ATCCT-positive alleles $(P<0.001)$.

We then examined the effects of the ATCCT repeat interruption on the correlation between age at onset and expansion size (Figure 3). We found a moderate, inverse correlation between age at onset and expansion size in ATCCT-negative individuals (Figure 3a; $\left.r=-0.5816, r^{2}=0.3383, P=0.002, n=35\right)$, and a non-significant inverse correlation in ATCCT-positive individuals (Figure 3b; $\left.r=-0.1936, r^{2}=0.03749, P=0.4271, n=19\right)$. 

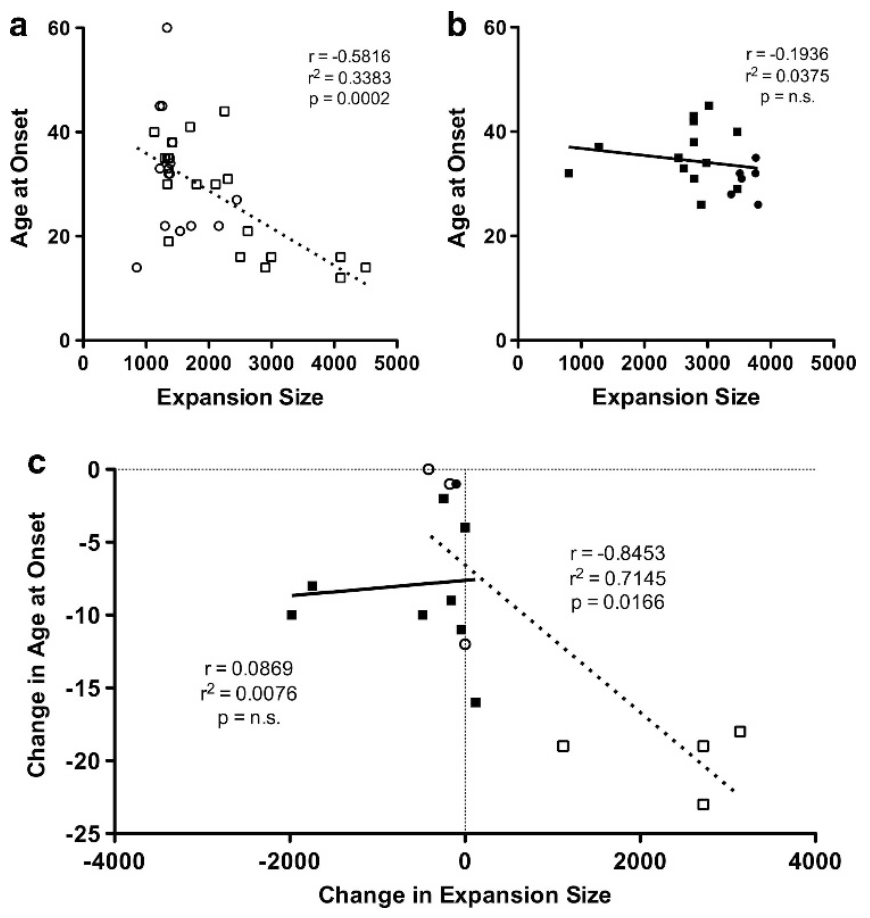

Figure 3 Effects of ATCCT interruptions on the relationship between expansion size and age at onset and genetic anticipation. (a) Age at onset plotted against expansion size for ATCCT-negative alleles (white circles, maternally transmitted alleles; white squares, paternally transmitted alleles, dashed line). (b) Age at onset plotted against expansion size for ATCCTpositive alleles (black circles, maternally transmitted alleles; black squares, paternally transmitted alleles, solid line). (c) Intergenerational changes in age at onset plotted against intergenerational changes in expansion size, symbols and lines as in panels $\mathbf{a}$ and $\mathbf{b}$.

To further examine the effects of ATCCT interruptions on the relationship between anticipation and intergenerational changes in expansion size, we plotted the change in age at onset against the change in expansion size in 16 parent-child pairs (Figure 3c). In ATCCT-negative transmissions, we find a strong, inverse correlation $(r=-0.8453$, $\left.r^{2}=0.7145, P=0.0166, n=7\right)$, indicating that as the expansion size increases in subsequent generations, the age at onset decreases. Conversely, for ATCCT-positive transmissions, we find a negligible, positive correlation $\left(r=0.08691, r^{2}=0.0076, P=0.8241, n=9\right)$.

\section{DISCUSSION}

In this study, we used a new assay to identify SCA10 expansions with ATCCT-interrupted motifs, and report on their effect on SCA10 expansion size, correlations between age at onset and expansion size as well as anticipation and intergenerational instability of the expansion. The ATCCT-PCR assay can rapidly determine the presence of ATCCT repeat interruptions within the 5 -end of SCA10 expansions. However, negative results from this assay need to be interpreted with caution as this may not necessarily indicate that an ATCCT-negative SCA10 expansion is a 'pure' or noninterrupted expansion. The SCA10 expansion may contain repeat interruptions, which yet give a negative result for a number of reasons including: (1) ATCCT-interrupted repeats are present but are farther interior, preventing amplification of a larger PCR product, (2) interrupted repeats contain heptanucleotide interruptions, but lack a sufficient length of ATCCT repeats for primer annealing, or (3) interrupted repeats of an entirely different motif are present as seen in a SCA10 patient with an unusually small, 280 repeat expansion allele and ATGCT interruptions. ${ }^{8}$

\section{ATCCT interruptions, age at onset and anticipation}

Many coding and noncoding repeat expansion disorders share the common genetic feature of anticipation, the phenomenon whereby symptoms appear at an earlier age than previous generations. Anticipation is explained by increases in repeat expansion size and a strong inverse correlation between repeat length and age at onset. In SCA10, anticipation as well as an inverse correlation between repeat length and age at onset was noted in Brazilian and some Mexican families. ${ }^{1,4,6}$ Yet, a separate Mexican-American family, where repeat interruptions were later identified, demonstrated anticipation but lacked the correlation between age at onset and repeat length. ${ }^{6,8,14}$ Our current study has confirmed this lack of correlation and has also demonstrated it in additional families. This study highlights the presence of ATCCT repeat interruptions as a contributing factor in these disparate results. For ATCCT-positive expansions, there is a statistically insignificant correlation between the age at onset and expansion size. Notably, for similarly sized alleles, ATCCT-positive expansions generally confer a later age at onset than those that are ATCCT negative. How can the presence of ATCCT interruptions counterbalance the effects of age at onset?

The majority of parent-child pairs we examined demonstrated anticipation, but to varying degrees (paternal, ATCCT-negative $>$ paternal, ATCCT-positive $>$ maternal, ATCCT-negative $>$ maternal, ATCCT-positive). However, only paternally transmitted, ATCCTnegative alleles demonstrated large gains in expansion size. This highlights a seemingly paradoxical effect of repeat interruptions on the relationship between anticipation and changes in expansion size between generations. Typically, decreasing the expansion size would be expected to have a beneficial effect by conferring a later age at onset in subsequent generations. However, in every ATCCT-positive transmission, but one, there is a decrease in expansion size with a concomitant decrease in the age at onset. Notably, in these ATCCTpositive transmissions, the child inherits the ATCCT interruption from the parent and is ATCCT positive. The molecular mechanisms of this paradox remain unknown. However, a small minority of CTG expanded alleles in myotonic dystrophy type 1 undergo a similar, paradoxical contraction process. ${ }^{15,16}$ How do ATCCT interruptions counteract the beneficial effects on the age at onset from decreasing the expansion size in subsequent generations?

\section{ATCCT interruptions and repeat instability}

The substantial difference on intergenerational repeat instability seen between paternally transmitted ATCCT-positive and ATCCT-negative expansions indicates possible differences in the underlying mechanism of repeat instability of these different alleles. A model might be proposed whereby strand slippage of complimentary strands of tandem repeats during DNA replication could generate instability of the SCA10 expansion. In such a model, ATATTCT, ATTTTCT and ATCCT repeat interruptions are then hypothesized to counteract strand slippage and act to stabilize the SCA10 expansions. This proposed mechanisms is similar to what was demonstrated for the CAT interruptions in SCA1 and AGG interruptions in FRAXA repeat expansions. ${ }^{17}$ Accordingly, the presence of AGG interruptions in maternal premutation alleles of the fragile X syndrome decreases the chance of expansion to a full mutation allele in their children. ${ }^{18}$ Such a mechanism may be in place for maternally transmitted, ATCCT-positive expansions. Although the sizable contractions (ATCCT-positive) and expansions (ATCCTnegative) of paternally transmitted expansions could still involve 
misaligned strands due to slippage, a different mechanism may also be considered. Such large changes in expansion size could come about via unequal crossing-over during genetic recombination as is proposed in the CCTG expansions from myotonic dystrophy type 2 . In this case, the 'crossover hotspot' may be a result of the low thermodynamic stability of the CCTG repeats. ${ }^{19}$ Along these lines, long normal ATTCT repeats appear to act as an in vitro DNA-unwinding element, ${ }^{20}$ which are thermodynamically unstable genomic regions that can support initiation of replication. ${ }^{21}$

\section{ATCCT interruptions and SCA10 expansion size}

SCA10 expansions containing ATCCT repeat interruptions tend to be longer than those without the interruptions. As paternal transmission of ATCCT-positive expansions results in large contractions in size, whereas maternal transmissions are relatively stable, this begs the questions of the origin of large SCA10 expansions with ATCCT interruptions. The common disease haplotype that exists between SCA10 families, including those that whose expansions do as well as do not carry ATCCT interruptions, supports the idea of a single origin of the expansion. ${ }^{22}$ Did the uninterrupted expansion occur first and then gather interruptions? Or did interruptions arise first and were later lost from large expansions? Large normal SCA10 alleles ( $\geq 17$ repeats) typically contain interrupted ATTGT or TTTCT motifs next to the most distal ATTCT motif, whereas smaller, normal alleles ( $\leq 16$ repeats) are uninterrupted. ${ }^{8}$ Such interruptions in large, normal alleles are thought to act to stabilize these alleles from further expansion, as is proposed for SCA1 and SCA2, where interruptions are also found in long, normal alleles but are absent from pathogenically expanded alleles. $^{23,24}$ However, the smallest disease-causing SCA10 allele (280 repeats) with reduced penetrance has multiple ATGCT interruptions, suggesting that the expansion origin of SCA10 repeat is not straightforward. ${ }^{8}$ Further studies will be needed to decipher the origin of SCA10 expansions.

Other effects of the repeat interruption on disease duration, age at death and other disease phenotypes remains unexplored. Why the ATCCT interruption can counterbalance the beneficial effect of a smaller expansion size on the age at onset is a question that may be explored by testing the hypothesis that interrupting sequences modify the pathogenic potency of the expanded alleles. As SCA10 is considered to act through an RNA mediated gain-of-function, ${ }^{11,25,26}$ further study on RNA toxicity of AUCCU-positive and -negative alleles may shed light on this question. Finally, it will be interesting to determine whether there are additional repeat interruptions in the expansions of other SCA10 patients.

\section{CONFLICT OF INTEREST}

The authors declare no conflict of interest.

\section{ACKNOWLEDGEMENTS}

The study was supported by NS041547 (TA).
1 Rasmussen A, Matsuura T, Ruano L et al: Clinical and genetic analysis of four Mexican families with spinocerebellar ataxia type 10. Ann Neurol 2001; 50: 234-239.

2 Matsuura T, Achari M, Khajavi M, Bachinski LL, Zoghbi HY, Ashizawa T: Mapping of the gene for a novel spinocerebellar ataxia with pure cerebellar signs and epilepsy. Ann Neurol 1999; 45: 407-411.

3 Gatto EM, Gao R, White MC et al: Ethnic origin and extrapyramidal signs in an Argentinean spinocerebellar ataxia type 10 family. Neurology 2007; 69: 216-218.

4 Teive HA, Roa BB, Raskin S et al: Clinical phenotype of Brazilian families with spinocerebellar ataxia 10. Neurology 2004; 63: 1509-1512.

5 Teive HA, Munhoz RP, Raskin S et al: Spinocerebellar ataxia type 10: Frequency of epilepsy in a large sample of Brazilian patients. Mov Disord 2010; 25: 2875-2878.

6 Grewal RP, Achari M, Matsuura T et al: Clinical features and ATTCT repeat expansion in spinocerebellar ataxia type 10. Arch Neurol 2002; 59: 1285-1290.

7 Matsuura T, Yamagata T, Burgess DL et al: Large expansion of the ATTCT pentanucleotide repeat in spinocerebellar ataxia type 10. Nat Genet 2000; 26: 191-194.

8 Matsuura T, Fang P, Pearson CE et al: Interruptions in the expanded ATTCT repeat of spinocerebellar ataxia type 10: repeat purity as a disease modifier? Am J Hum Genet 2006; 78: 125-129.

9 Wang JL, Wu YQ, Lei LF et al: Polynucleotide repeat expansion of nine spinocerebellar ataxia subtypes and dentatorubral-pallidoluysian atrophy in healthy Chinese Han population. Zhonghua Yi Xue Yi Chuan Xue Za Zhi 2010; 27: 501-505.

10 Raskin S, Ashizawa T, Teive HA et al: Reduced penetrance in a Brazilian family with spinocerebellar ataxia type 10. Arch Neurol 2007; 64: 591-594.

11 Wakamiya M, Matsuura T, Liu Y et al: The role of ataxin 10 in the pathogenesis of spinocerebellar ataxia type 10. Neurology 2006; 67: 607-613.

12 Cagnoli C, Michielotto C, Matsuura T et al: Detection of large pathogenic expansions in FRDA1, SCA10, and SCA12 genes using a simple fluorescent repeat-primed PCR assay. J Mol Diagn 2004; 6: 96-100.

13 Matsuura T, Ashizawa T: Polymerase chain reaction amplification of expanded ATTCT repeat in spinocerebellar ataxia type 10. Ann Neurol 2002; 51 271-272.

14 Matsuura T, Fang P, Lin X et al: Somatic and germline instability of the ATTCT repeat in spinocerebellar ataxia type 10. Am J Hum Genet 2004; 74: 1216-1224.

15 Ashizawa $\mathrm{T}$, Anvret $\mathrm{M}$, Baiget $\mathrm{M}$ et al: Characteristics of intergenerationa contractions of the CTG repeat in myotonic dystrophy. Am J Hum Genet 1994; 54 414-423.

16 Monckton DG, Wong LJ, Ashizawa T, Caskey CT: Somatic mosaicism, germline expansions, germline reversions and intergenerational reductions in myotonic dystrophy males: small pool PCR analyses. Hum Mol Genet 1995; 4: 1-8.

17 Pearson CE, Eichler EE, Lorenzetti D et al: Interruptions in the triplet repeats of SCA1 and FRAXA reduce the propensity and complexity of slipped strand DNA (S-DNA) formation. Biochemistry 1998; 37: 2701-2708.

18 Yrigollen CM, Durbin-Johnson B, Gane L et al: AGG interruptions within the materna FMR1 gene reduce the risk of offspring with fragile X syndrome. Genet Med 2012; 14 729-736.

19 Dere R, Wells RD: DM2 $\mathrm{CCTG}^{*} \mathrm{CAGG}$ repeats are crossover hotspots that are more prone to expansions than the DM1 CTG*CAG repeats in Escherichia coli. J Mol Biol 2006; 360: 21-36

20 Liu G, Bissler JJ, Sinden RR, Leffak M: Unstable spinocerebellar ataxia type 10 (ATTCT*(AGAAT) repeats are associated with aberrant replication at the ATX10 locus and replication origin-dependent expansion at an ectopic site in human cells. $\mathrm{Mol} \mathrm{Cel}$ Biol 2007; 27: 7828-7838.

21 Kowalski D, Eddy MJ: The DNA unwinding element: a novel, cis-acting component that facilitates opening of the Escherichia coli replication origin. EMBO J 1989; 8 4335-4344.

22 Almeida T, Alonso I, Martins $\mathrm{S}$ et al: Ancestral origin of the ATTCT repeat expansion in spinocerebellar ataxia type 10 (SCA10). PLoS One 2009; 4: e4553.

23 Chung MY, Ranum LP, Duvick LA, Servadio A, Zoghbi HY, Orr HT: Evidence for a mechanism predisposing to intergenerational CAG repeat instability in spinocerebellar ataxia type I. Nat Genet 1993; 5: 254-258.

24 Pulst SM, Nechiporuk A, Nechiporuk T et al: Moderate expansion of a normally biallelic trinucleotide repeat in spinocerebellar ataxia type 2. Nat Genet 1996; 14 269-276.

25 White M, Xia G, Gao R et al: Transgenic mice with SCA10 pentanucleotide repeats show motor phenotype and susceptibility to seizure: A toxic RNA gain-of-function model. J Neurosci Res 2012: 90: 706-714.

26 White MC, Gao R, Xu W et al: Inactivation of hnRNP K by expanded intronic AUUCU repeat induces apoptosis via translocation of PKCdelta to mitochondria in spinocerebellar ataxia 10. PLoS Genet 2010; 6: e1000984.

Supplementary Information accompanies this paper on European Journal of Human Genetics website (http://www.nature.com/ejhg) 